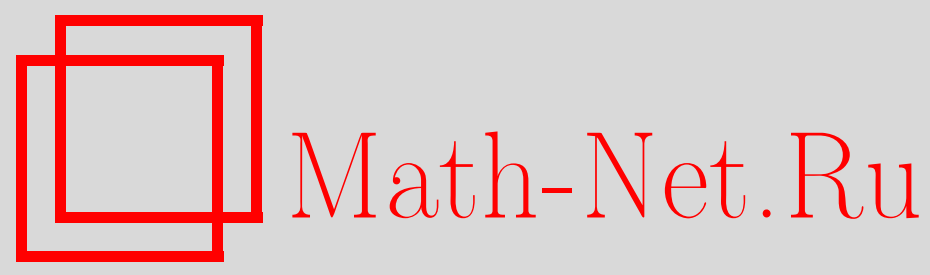

В. Ф. Гапошкин, Оценки энтропии множества средних некоторых классов стационарных и квазистационарных последовательностей, Матем. заметки, 2005, том 78, выпуск $1,52-58$

DOI: https://doi.org/10.4213/mzm2561

Использование Общероссийского математического портала Math-Net.Ru подразумевает, что вы прочитали и согласны с пользовательским соглашением http://www . mathnet.ru/rus/agreement

Параметры загрузки:

IP: 54.196.121.252

26 апреля 2023 г., 15:56:57

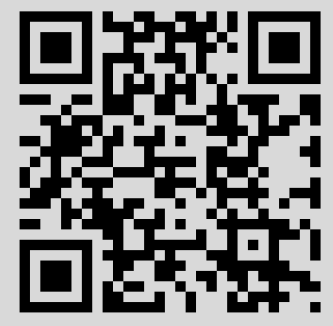




\section{ОЦЕНКИ ЭНТРОПИИ МНОЖЕСТВА СРЕДНИХ НЕКОТОРЫХ КЛАССОВ СТАЦИОНАРНЫХ И КВАЗИСТАЦИОНАРНЫХ ПОСЛЕДОВАТЕЛЬНОСТЕЙ}

\section{В. Ф. Гапошкин}

Рассматривается множество средних ариффметических некоторых классов стационарных и квазистационарных последовательностей. Приводятся оценки энтропии этого множества, точные по порядку в рассматриваемых классах.

Библиографиял: 5 названий.

Пусть $\left(X_{k}\right)_{k=1}^{\infty}-$ последовательность случайных величин в $L^{2}(\Omega) ;\|X\|=\left(\mathrm{E}|X|^{2}\right)^{1 / 2}$, $A=\left(A_{n}\right)$ - последовательность их средних арифметических,

$$
A_{n}=n^{-1} \sum_{k=1}^{n} X_{k}, \quad n=1,2, \ldots
$$

Если $\left(X_{k}\right)$ - стационарная в широком смысле последовательность, или $X_{k}=U^{k-1} f$, $f \in L^{2}, U$ - изометрический оператор, то по статистической эргодической теореме последовательность $A_{n}$ всегда сходится по норме.

В работах [1]-[3] исследовался порядок метрической энтропии $N(\varepsilon)$ предкомпактного множества $A=\left(A_{n}\right)_{n=1}^{\infty}$, где при $\varepsilon>0 N(\varepsilon)$ - наименьшее число замкнутых шаров радиуса $\varepsilon$ в $L^{2}(\Omega)$, покрывающих $A$. В работах [1], [2] показано, что для любых $f \in L^{2}$ и $U$ справедлива оценка

$$
N(\varepsilon)=O\left(\varepsilon^{-2}\right) \quad \text { при } \varepsilon \rightarrow 0 .
$$

Кроме того, имеются примеры пар $(f, U)$, для которых

$$
N(\varepsilon) \geqslant c_{0} \varepsilon^{-2}, \quad c_{0}>0 .
$$

В работе [3] получен следующий результат. Пусть стационарная последовательность $\left(X_{k}\right)$ удовлетворяет при некотором $\alpha>0$ условию

$$
\int_{0<|\lambda|<\lambda_{0}}\left(\lg \frac{1}{|\lambda|}\right)^{\alpha} d F(\lambda)<\infty,
$$

где $d F(\lambda)$ - спектральная мера последовательности. Тогда справедлива оченка

$$
N(\varepsilon)=O\left(\varepsilon^{-2}\left(\lg \frac{1}{\varepsilon}\right)^{-\alpha}\right) \quad \text { при } \varepsilon \rightarrow 0 .
$$


Естественно рассмотреть и другие классы стационарных последовательностей, заданные некоторыми ограничениями на их спектральную меру или корреляционную функцию. Далее предполагается, что $\left(X_{k}\right)$ - некоторая последовательность случайных величин из $L^{2}(\Omega)$, для которой выполнено следующее условие квазистационарности: при некоторых $C_{0}>0,0<\alpha \leqslant 2$

$$
\left\|n^{-1} \sum_{k=m+1}^{m+n} X_{k}\right\|^{2} \leqslant C_{0}^{2} n^{-\alpha}, \quad \forall m \geqslant 0, \quad n \geqslant 1 .
$$

Если $\left(X_{k}\right)$ - стационарная последовательность, то при оценке энтропии можно считать, не уменьшая общности, что $\mathrm{E} X_{k}=0$. Пусть $K(n)$ - коррелящионная функция, $K(n)=\mathrm{E} X_{k} X_{k+n}, k=1,2, \ldots, n=1,2, \ldots$ Ограничение (4) тесно связано с аналогичными ограничениями на $K(n)$ или спектральную меру $d F(\lambda)$. Известны следующие утверждения (см., например, [4], [5]).

ЛЕмма 1. А) Если при некотором $0<\alpha<1$ и $C_{1}>0$ выполнено условие

$$
|K(n)| \leqslant C_{1} n^{-\alpha}
$$

то выполнено условие (4).

Б) Если при некоторых $0<\alpha<1 u \lambda_{0}>0$

$$
\int_{0<|\lambda|<\lambda_{0}}|\lambda|^{-\alpha} d F(\lambda)<\infty, \quad F(+0)-F(-0)=0
$$

то выполнено условие (4).

В) Если для стационарной последовательности выполнено условие (4) при некотором $\alpha>2$, то $X_{k}=0, k=1,2, \ldots$, почти всюду.

ТЕОРемА 1. Пусть выполнено условие квазистационарности (4). Тогда для әнтропии $N(\varepsilon)$ множества средних справедливо неравенство

$$
N(\varepsilon) \leqslant C_{0} D_{\alpha} \varepsilon^{-1}
$$

Постоянные $D_{\alpha}$ допускают оиенку

$$
D_{\alpha} \leqslant\left(\frac{2-\alpha}{\alpha}\right)^{(2-\alpha) / \alpha}+2 \quad \text { npu } 0<\alpha<1, \quad D_{\alpha} \leqslant 3 \quad \text { npu } 1 \leqslant \alpha \leqslant 2 .
$$

Таким образом, даже при близких к нулю значениях $\alpha$ порядок по $\varepsilon$ верхней оценки энтропии в соответствующем классе стационарных или квазистационарных последовательностей существенно улучшается по сравнению с общей оценкой (1).

Отметим также, что доказательства оценок $(1),(3)$ были достаточно сложньми. Они использовали тонкие оценки стохастической спектральной меры стационарной последовательности и метод регуляризации этой меры. В то же время доказательство теоремы 1 использует только оценки норм $\left\|A_{n}-A_{m}\right\|$ и поэтому проходит также и для квазистационарных последовательностей. 
ЗАмЕЧАниЕ 1. Если условие (4) выполнено при некотором $\alpha_{1}>0$, то оно выполнено и при всех $\alpha>\alpha_{1}$. Поэтому $D_{\alpha} \leqslant D_{1}$ при $1<\alpha \leqslant 2$, и достаточно доказать теорему 1 в случае $0<\alpha \leqslant 1$.

Лемма 2. Пусть при некотором $0<\alpha \leqslant 1$ выполнено условие (4). Тогда

$$
\left\|A_{n}-A_{m}\right\|^{2} \leqslant \rho^{2}(n, m) \quad n p u \quad 1 \leqslant m<m,
$$

$2 \partial e$

$$
\rho^{2}(n, m)=2^{2-\alpha} C_{0}^{2} \frac{(n-m)^{2-\alpha}}{n^{2-\alpha} m^{\alpha}} .
$$

ДокАЗАТЕЛЬСТво. Запишем при $n>m$

$$
A_{n}-A_{m}=\left(\frac{1}{n}-\frac{1}{m}\right) \sum_{k=1}^{m} X_{k}+\frac{1}{n} \sum_{k=m+1}^{n} X_{k}
$$

откуда

$$
\left\|A_{n}-A_{m}\right\|^{2} \leqslant 2 C_{0}^{2}\left[\frac{(n-m)^{2}}{n^{2} m^{2}} m^{2-\alpha}+\frac{1}{n^{2}}(n-m)^{2-\alpha}\right] .
$$

Используя неравенство $m^{\alpha}+(n-m)^{2-\alpha} \leqslant 2^{1-\alpha} n^{\alpha}$, получим $(6),(7)$.

Пусть

$$
0<\beta \leqslant 1
$$

тогда $B=\beta^{-1} \geqslant 1$. Положим $\alpha_{0}=1$ и определим последовательно $a_{1}, a_{2}, \ldots$ из уравнений

$$
a_{k+1}\left(1-a_{k+1}^{\beta}\right)^{-1}=a_{k}, \quad k=0,1, \ldots
$$

Очевидно, что на каждом шаге решения $a_{1}, a_{2}, \ldots$ определяются однозначно, $0<$ $a_{k}<1$ и и $a_{k+1}<a_{k}, k=0,1, \ldots$

Лемма 3. Справедливы оченки

$$
\frac{1}{(k+1)^{B}} \leqslant a_{k} \leqslant\left(\frac{B}{B+k}\right)^{B}, \quad k=0,1, \ldots
$$

ДокАЗАТЕЛЬСТво. Положим $b_{k}=a_{k}^{B}$; тогда $a_{k}=b_{k}^{\beta}$ и нужно проверить неравенства

$$
\frac{1}{k+1} \leqslant b_{k} \leqslant \frac{B}{B+k}, \quad k=0,1, \ldots .
$$

Применим индукцию. При $k=0$ эти соотношения выполнены. Равенство (9) переписывается в виде $b_{k+1}=\left(1-b_{k+1}\right)^{\beta} b_{k}$. Так как $0<\beta \leqslant 1$, то

$$
\left(1-b_{k+1}\right) b_{k} \leqslant b_{k+1} \leqslant\left(1-b_{k+1} \beta\right) b_{k} .
$$

Если $b_{k} \leqslant B /(B+k)$, то

$$
b_{k+1} \leqslant\left(1-b_{k+1} \beta\right) \frac{B}{B+k}, \quad \text { т.e. } \quad b_{k+1} \leqslant \frac{B}{B+k+1} .
$$


Аналогично, если $b_{k} \geqslant(k+1)^{-1}$, то

$$
b_{k+1} \geqslant\left(1-b_{k+1}\right) \frac{1}{k+1} \quad \text { и } \quad b_{k+1} \geqslant \frac{1}{k+2} .
$$

Лемма 3 доказана.

Далее удобно считать, что функция $\rho^{2}(n, m)$ задается формулой $(7)$ при любых действительных $n>m>0$. Пусть выполнено предположение (4). Будем считать, что

$$
0<\alpha \leqslant 1, \quad \varepsilon>0, \quad \beta=\frac{\alpha}{2-\alpha} \leqslant 1, \quad B=\beta^{-1}, \quad n_{0}=\varepsilon^{-(\beta+1) / \beta} .
$$

Найдем рекуррентно положительные числа $n_{1}, n_{2}, \ldots$ из уравнений

$$
n_{k}=\frac{n_{k+1}}{1-\varepsilon^{\beta+1} n_{k+1}^{\beta}} .
$$

\section{Лемма 4. При сделанных предположениях}

1) числа $n_{k}$ однозначно определяются из уравнений (12);

2) $n_{k}=a_{k} \varepsilon^{-(\beta+1) / \beta}$, где монотонно убивающие числа а уравнениям (9), $a_{0}=1$;

3) при $n \in\left[n_{k}, n_{k-1}\right]$ справедлива оченка

$$
\rho^{2}\left(n, n_{k}\right) \leqslant 2^{2-\alpha} C_{0}^{2} \varepsilon^{2}
$$

ДокАЗАТЕЛЬСТВо. Если обозначить $a_{0}=1, a_{k}=\varepsilon^{(\beta+1) / \beta} n_{k}$, то числа $a_{k}$ будут удовлетворять уравнению (9) и утверждения 1) и 2) следуют из леммы 3.

Утверждение 3) означает, что

$$
\frac{\left(n-n_{k}\right)^{2-\alpha}}{n^{2-\alpha} n_{k}^{\alpha}} \leqslant \varepsilon^{2} \quad \text { или } \quad n-n_{k} \leqslant \varepsilon^{\beta+1} n_{k}^{\beta},
$$

что верно при $n \in\left[n_{k}, n_{k-1}\right]$.

ДокАЗАТЕльство теоремы 1 . Далее $K(0, \varepsilon)$ - замкнутый шар в $L^{2}$ радиуса $\varepsilon>0$ с центром в нуле, $K(l, \varepsilon)$ - замкнутые шары радиуса $\varepsilon$ с центрами в $A_{l}, l=1,2, \ldots$. Пусть числа $\alpha, \beta, \varepsilon, B, n_{0}$ удовлетворяют (11). Положим $\varepsilon_{1}=2^{(2-\alpha) / 2} C_{0} \varepsilon$ и построим $\varepsilon_{1}$-сеть для $A=\left(A_{n}\right)_{n=1}^{\infty}$ следуюшим образом. Так как $n_{0}=\varepsilon^{-(\beta+1) / \beta}$, то при $n \geqslant n_{0}$

$$
\left\|A_{n}\right\|^{2} \leqslant C_{0}^{2} n^{-\alpha} \leqslant C_{0}^{2} \varepsilon^{2}
$$

т.е. $\left\{A_{n} ; n \geqslant n_{0}\right\} \subset K\left(0, \varepsilon_{1}\right)$. Далее интересен лишь случай $0<\varepsilon<1$, когда $n_{0}>1$. Образуем по формулам (12) действительные числа $n_{1}, n_{2}, \ldots$ По лемме 4 при $n \in\left[n_{k}, n_{k-1}\right]$

$$
\rho^{2}\left(n, n_{k}\right) \leqslant 2^{2-\alpha} C_{0}^{2} \frac{\left(n-n_{k}\right)^{2-\alpha}}{n^{2-\alpha} n_{k}^{\alpha}} \leqslant \varepsilon_{1}^{2} .
$$


Числа $n_{k}$ могут оказаться не целыми. Положим $s=\left[\varepsilon^{-1}\right]$ и $m_{k}=n_{k}$ при $n_{k}$ целом, $m_{k}=\left[n_{k}\right]+1, k=1,2, \ldots, s$, при $n_{k}$ не целом. Образуем множество $D=\left\{0, A_{1}, A_{2}, \ldots\right.$, $\left.A_{m_{s}-1}, A_{m_{1}}, A_{m_{2}}, \ldots, A_{m_{s}}\right\}$. Так как $B+s \geqslant \varepsilon^{-1}$, то

$$
m_{s} \leqslant n_{s}+1 \leqslant\left(\frac{B}{B+s}\right)^{B} \varepsilon^{-B-1}+1 \leqslant B^{B} \varepsilon^{-1}+1 .
$$

Значит, $\operatorname{card} D=m_{s}+s \leqslant\left(B^{B}+1\right) \varepsilon^{-1}+1$. Покажем, что $D$ является $\varepsilon_{1}$-сетью для $A=\left(A_{n}\right)$. Рассмотрим любое целое $n, n_{s} \leqslant n<n_{0}$. Тогда при некотором $k, 1 \leqslant k \leqslant s$, имеем $n_{k} \leqslant n<n_{k-1}$. Так как

$$
\frac{\left(n-m_{k}\right)^{2-\alpha}}{n^{2-\alpha} m_{k}^{\alpha}} \leqslant \frac{\left(n-n_{k}\right)^{2-\alpha}}{n^{2-\alpha} n_{k}^{\alpha}},
$$

из (13) следует, что $\rho^{2}\left(n, m_{k}\right) \leqslant \varepsilon_{1}^{2}$, т.е. $A_{n} \in K\left(m_{k}, \varepsilon_{1}\right)$. Итак, любой элемент $A_{n}$ входит либо в шар $K\left(0, \varepsilon_{1}\right)$, если $n \geqslant n_{0}$, либо в какой-то шар $K\left(m_{k}, \varepsilon_{1}\right), 1 \leqslant k \leqslant s$, если $m_{k} \leqslant n<n_{0}$, либо является центром шара $K\left(n, \varepsilon_{1}\right)$, если $1 \leqslant n<m_{k}$. Значит,

$$
N\left(\varepsilon_{1}\right) \leqslant \operatorname{card} D \leqslant\left(B^{B}+1\right) \varepsilon^{-1}+1 .
$$

Так как эта оценка справедлива при любом $\varepsilon>0$, а $\varepsilon_{1}=2^{(2-\alpha) / 2} C_{0} \varepsilon$, то

$$
N(\varepsilon) \leqslant\left(B^{B}+1\right) 2^{(2-\alpha) / 2} C_{0} \varepsilon^{-1}+1 .
$$

Теорема 1 доказана.

Следуюшие два примера показывают точность оценки теоремы 1 (по порядку $\varepsilon$ при $\varepsilon \rightarrow 0)$. Если $\left(X_{k}\right)$ - ортонормированная система (ОНС) в $L^{2}(\Omega)$, то при всех $n, m \geqslant 1$ имеем $\left\|n^{-1} \sum_{k=m+1}^{m+n} X_{k}\right\|^{2}=n^{-1}$, т.е. вьполнено неравенство (4) при $\alpha=1$.

ПРИМЕР 1 [2, с. 105]. Для любой ОНС справедливы оценки энтропии средних $C_{1} \varepsilon^{-1} \leqslant N(\varepsilon) \leqslant C_{2} \varepsilon^{-1}$ при некоторых $C_{1}, C_{2}>0$. Константы нетрудно оценить, а именно,

$$
\varepsilon^{-1}-1 \leqslant N(\varepsilon) \leqslant\left[2 \varepsilon^{-1}\right]+1 \quad \text { при } \varepsilon>0 .
$$

Рассмотрим теперь крайний случай, когда стационарная последовательность $\left(X_{k}\right)$ удовлетворяет условию (4) с максимально возможньм показателем $\alpha=2$. Как известно, пример такой последовательности может быть построен в виде

$$
X_{k}=\frac{Y_{k}-Y_{k+1}}{\sqrt{2}}, \quad k=1,2, \ldots
$$

где $\left(Y_{k}\right)$ - некоторая ОНС. Тогда

$$
A_{n}=\frac{Y_{1}-Y_{n+1}}{n \sqrt{2}}, \quad\left\|A_{n}\right\|^{2}=n^{-2} .
$$


ПримеР 2. Для этой стационарной последовательности $\left(X_{k}\right)$ справедливы оценки энтропии

$$
\left[(\sqrt{2} \varepsilon)^{-1}\right] \leqslant N(\varepsilon) \leqslant\left[\varepsilon^{-1}\right]+1 .
$$

ДоКАЗАТЕЛЬСтво. Имеем

$$
2\left\|A_{n}-A_{m}\right\|^{2}=\left(\frac{1}{n}-\frac{1}{m}\right)^{2}+\frac{1}{n^{2}}+\frac{1}{m^{2}} .
$$

Поэтому если $m \leqslant(\sqrt{2} \varepsilon)^{-1}$, а $n$ любое, то $\left\|A_{n}-A_{m}\right\|>\varepsilon$. Следовательно, $N(\varepsilon) \geqslant$ $\left[(\sqrt{2} \varepsilon)^{-1}\right]$. С другой стороны, при $n>\varepsilon^{-1}\left\|A_{n}\right\|^{2} \leqslant n^{-2}<\varepsilon^{2}$, т.е. $\left\{A_{n} ; n>\varepsilon^{-1}\right\} \subset$ $K(0, \varepsilon)$, и множество $D=\left\{0, A_{1}, A_{2}, \ldots, A_{n_{0}}\right\}$ образует $\varepsilon$-сеть для $A, n_{0}=\left[\varepsilon^{-1}\right]$. Следовательно, $N(\varepsilon) \leqslant n_{0}+1=\left[\varepsilon^{-1}\right]+1$.

Любопытно, что существуют нетривиальные стационарные последовательности, для которых $N(\varepsilon)=o\left(\varepsilon^{-1}\right)$ при $\varepsilon \rightarrow+0$. Пусть $X_{1}$ - некоторая случайная величина, и

$$
\mathrm{E} X_{1}=0, \quad\left\|X_{1}\right\|=1, \quad X_{k}=(-1)^{k} X_{1}, \quad k=1,2, \ldots .
$$

Эта последовательность является стационарной, ее корреляционная функция имеет вид $K(n)=(-1)^{n}, n=0,1,2, \ldots$

ПримеР 3. Для последовательности (14) энтропия множества средних $A$ имеет порядок $O\left(\varepsilon^{-1 / 2}\right)$, точнее,

$$
\left[(2 \varepsilon)^{-1 / 2}\right] \leqslant N(\varepsilon) \leqslant 2\left[\varepsilon^{-1 / 2}\right]
$$

ДоказАтельство. Средние $A_{n}$ имеют вид $A_{2 n}=0, A_{2 n-1}=(2 n-1)^{-1} X_{1} \cdot \mathrm{B}$ частности, $\left(X_{n}\right)$ удовлетворяет условию (4) при $\alpha=2$. Очевидно, что энтропия одномерного множества $\left(A_{n}\right)$ в $L^{2}(\Omega)$ совпадает с энтропией числовой последовательности $(0,1,1 / 3,1 / 5, \ldots)$ на $[0,1]$. Поэтому оценки $(15)$ вытекают из следующей леммы.

Лемма 5. Пусть $a_{n}=n^{-1}, b_{n}=(2 n-1)^{-1}, n=1,2, \ldots u E=\left\{0, a_{n}\right\}_{n=1}^{\infty}$, $F=\left\{0, b_{n}\right\}_{n=1}^{\infty}-$ соответствующие компактные множества на отрезке [0,1]. Энтропия этих мнохеств при $0<\varepsilon<1$ удовлетворяет неравенствам

$$
\left[\varepsilon^{-1 / 2}\right]-1 \leqslant N_{E}(\varepsilon) \leqslant 2 \varepsilon^{-1 / 2}, \quad(2 \varepsilon)^{-1 / 2} \leqslant N_{F}(\varepsilon) \leqslant 2 \varepsilon^{-1 / 2} .
$$

Так как в литературе это простое утверждение нам не встречалось, приведем его доказательство.

ДокАЗАтЕЛЬСтво. Оценим $N_{E}(\varepsilon)=N(\varepsilon)$. Можно считать, что $0<\varepsilon<1 / 4$, так как при $\varepsilon \geqslant 1 / 4$ множество $E$ содержится в двух " $\varepsilon$-шарах" $[0,1 / 2]$ и $[1 / 2,1], 1 \leqslant$ $N(\varepsilon) \leqslant 2$. Так как $a_{n}-a_{n+1}>(n+1)^{-2} \geqslant \varepsilon$ при $1 \leqslant n \leqslant n_{0}(\varepsilon), n_{0}(\varepsilon)=\left[\varepsilon^{-1 / 2}\right]$, то элементы множества $\left\{a_{0}, a_{1}, \ldots, a_{n_{0}-1}\right\}$ являются $\varepsilon$-изолированными и $N(\varepsilon) \geqslant\left[\varepsilon^{-1 / 2}\right]-1$. Остальные элементы множества $A$ расположены на отрезке $\left[0, n_{0}^{-1}\right], \varepsilon$-энтропия которого не превосходит $\left(2 \varepsilon n_{0}\right)^{-1}$. Следовательно,

$$
N(\varepsilon) \leqslant n_{0}-1+\left(2 \varepsilon n_{0}\right)^{-1} \leqslant 2 \varepsilon^{-1 / 2} .
$$

Оценка $N_{F}(\varepsilon)$ проходит аналогично; она использует неравенство

$$
b_{n}-b_{n+1}=\frac{2}{4 n^{2}-1}>\varepsilon \quad \text { при } n \leqslant(2 \varepsilon)^{-1 / 2} .
$$


ЗАмЕчАниЕ 2. Аналогично доказывается, что при любом $\alpha>0$ энтропия множества $\left\{n^{-\alpha} ; n \geqslant 1\right\} \subset[0,1]$ имеет порядок $O\left(\varepsilon^{-\alpha /(\alpha+1)}\right)$ при $\varepsilon \rightarrow 0$.

Отметим два нерешенных вопроса.

ГипотезА 1. Не существует стационарной последовательности $\left(X_{k}\right)_{k=1}^{\infty}, X_{k} \neq X_{1}$ при $k \geqslant 2$, для которой множество средних арифметических имеет энтропию $N(\varepsilon)=$ $o\left(\varepsilon^{-1 / 2}\right)$ при $\varepsilon \rightarrow 0$.

ГипотезА 2. Не существует стационарной последовательности $\left(X_{k}\right)_{k=1}^{\infty}, X_{k} \neq X_{1}$ при $k \geqslant 2$, для которой $N(\varepsilon)$ имеет точный порядок $O\left(\varepsilon^{-\beta}\right), 1 / 2<\beta<1$.

ЗАМЕчАНИЕ 3. Конечно, квазистационарные последовательности со сколь угодно медленной скоростью роста энтропии при $\varepsilon \rightarrow 0$ существуют.

Например, легко построить последовательность случайных величин, для которых при фиксированном $\alpha>2$ выполнено соотношение $\left\|A_{n}\right\|^{2} \leqslant n^{-\alpha}$. Тогда $N(\varepsilon) \leqslant \varepsilon^{-2 / \alpha}$. Это следует из оценки $\left\|A_{n}\right\|^{2} \leqslant n^{-\alpha} \leqslant \varepsilon^{2}$ при $n \geqslant n(\varepsilon)$, где $n(\varepsilon)=\left[\varepsilon^{-2 / \alpha}\right]-1$, и $\varepsilon$-сеть для $A=\left(A_{n}\right)$ имеет вид $\left\{0, A_{1}, A_{2}, \ldots, A_{n(\varepsilon)-1}\right\}$.

Автор благодарен рецензенту за полезные редакционные замечания.

\section{СПИСОК ЦИТИРОВАННОЙ ЛИТЕРАТУРЫ}

[1] Talagrand M. Applying a theorem of Fernique // Ann. Inst. H. Poincaré. 1996. V. 32. P. 779-799.

[2] Weber M. Entropie metrique et convergence presque partout. Paris: Hermann, 1998.

[3] Gamet C., Weber M. Entropy numbers of some ergodic averages // Теор. вероятн. и еe примен. 1999. Т. 44. № 4. С. 776-795.

[4] Гапошкин В. Ф. Сходимость рядов, связанных со стационарньми последовательностями // Изв. АН СССР. Сер. матем. 1975. Т. 39. №6. С. 1365-1392.

[5] Гапошкин В.Ф. О скорости убывания вероятностей $\varepsilon$-уклонений средних стационарных процессов // Матем. заметки. 1998. Т. 64. № 3. С. 366-372. 